\title{
Evaluation of Antimicrobial Susceptibility Among Enterococcus Species by E-Test Method at Khatam-ol-Anbia Hospital During 2013-2014
}

\author{
Davood Yadegarynia ; Sara Rahmati Roodsari ${ }^{1}$ and Zahra Arab-Mazar," \\ ${ }^{1}$ Infectious Diseases and Tropical Medicine Research Center, Shahid Beheshti University of Medical Sciences, Tehran, IR Iran \\ ${ }^{*}$ Corresponding author: Zahra Arab-Mazar, Infectious Diseases and Tropical Medicine Research Center, Shahid Beheshti University of Medical Sciences, Tehran, IR Iran. Tel: \\ +98-2122439963, Fax: +982122439964, E-mail: arabmazar@sbmu.ac.ir; z.arabmazar@gmail.com
}

Received 2015 May 9; Revised 2015 October 3; Accepted 2015 October 5.

\begin{abstract}
Background: Over the past decade, Enterococci have been shown to be an important cause of nosocomial and community-acquired infections. Inappropriate use of antibiotics led to changes in the pattern of antibiotic resistances in Enterococcus species. Unfortunately, no study has been performed in Iran in recent years regarding the antimicrobial resistance of Enterococci using the E-test method as a base. We must gain sufficient knowledge about the regional antibiotic resistances related to Enterococcus so that we can monitor the prevalence and antimicrobial resistance of Enterococcus by administering appropriate treatments.

Objectives: The objective of this study was to evaluate the antimicrobial susceptibility among Enterococcus species by the E-test method at Khatam-ol-Anbia hospital during 2013-2014.

Patients and Methods: This descriptive cross-sectional study was carried out during 2013-2014. All clinical samples were collected from the intensive care unit(ICU) and general wards of Khatam-ol-Anbia hospital. All Enterococcus species were detected via biochemical testing. Antimicrobial susceptibility and minimum inhibitory concentration (MIC) were determined via disk diffusion and the E-test method. We used descriptive statistics to analyze the data.

Results: A total of 53 Enterococci were isolated from clinical samples of blood, urine, wounds, sputum, and cerebrospinal fluid (CSF) over a two-year period from the ICU and general wards. The isolated Enterococcus species were $77.35 \%$ E. faecalis, $18.86 \%$ E. faecium, and $3.77 \%$ other species. Species evaluated by E-test were resistant to imipenem, ampicillin, co-trimoxazole, gentamicin, rifampicin, vancomycin, linezolid, and teicoplanin; $54 \%, 68 \%, 100 \%, 93.8 \%, 60.4 \%, 39.6 \%, 0 \%$, and $29.2 \%$, respectively. Among the strains of enterococci, 90.9\% of E. faecium and $20 \%$ of $E$. faecalis species were resistant to vancomycin.

Conclusions: According to these findings, antibiotic-resistance patterns have changed, and vancomycin resistance, especially among $E$. faecium, is rising because of nosocomial infections. Consequently, it has become a serious subject for patients admitted into a hospital.
\end{abstract}

Keywords: Microbial Sensitivity Tests, E-test, Enterococcus

\section{Background}

Over the past decade, enterococci have become an important cause of nosocomial and community-acquired infections. Also, in the United States, enterococci are considered to be the second most common cause of nosocomial infections. Most infections caused by these pathogens are urinary tract infections, intra-abdominal and pelvic abscesses, biliary infections, surgical wound infections, bacteremia, infections of the central nervous system (CNS), neonatal infection, and in rare instances respiratory, osteomyelitis, and cellulitis (1).

In recent years, the extensive use of intravascular devices, prosthesis, cytotoxic chemotherapy, and immune deficiency drugs have increased the importance of these micro-organisms. In recent studies, it has been reported that such infections are transmitted via organ transplantation $(2,3)$.

Resistance to antibiotics is characteristic of enterococci, whereas some species, such as E. faecium, have shown more resistance (4). In the survey, the resistance pattern shows evidence of changing due to inappropriate therapy. In recent studies, antibiotics such as daptomycin and linezolid have been proposed instead of vancomycin (5). The clinical and laboratory standards institute (CLSI) suggests the E-test method is superior to the antibiotic resistances (6). Unfortunately, in recent years, there have not been any comprehensive studies to assess the resistances of enterococci via E-test.

\section{Objectives}

This study was performed in order to investigate the antibiotic resistances in enterococci by using E-test at the Khatam-ol-Anbia hospital during 2013 - 2014. To deal with the aforementioned issues, our objective was to learn about the antibiotic resistances in every region, choose the correct treatment, and take the necessary steps to

Copyright (C) 2016, Infectious Diseases and Tropical Medicine Research Center. This is an open-access article distributed under the terms of the Creative Commons Attribution-NonCommercial 4.0 International License (http://creativecommons.org/licenses/by-nc/4.0/) which permits copy and redistribute the material just in noncommercial usages, provided the original work is properly cited. 
prevent further resistances, thereby reducing morbidity and mortality.

\section{Patients and Methods}

This was a cross-sectional study. During the project, samples containing isolated Enterococcus were sent from different parts of the Khatam-ol-Anbia hospital in Tehran, Iran during 2013 - 2014. The samples were inoculated onto Enterococcosel agar after 24 hours of incubation at $37^{\circ} \mathrm{C}$. Isolates were confirmed to be enterococci by Gram stain, pyrrolidonyl arylamidase activity, motility, and catalase, and were then subcultured onto three culture media: Mueller-Hinton agar to determine their growth at $15^{\circ} \mathrm{C}$ and $45^{\circ} \mathrm{C}, \mathrm{NaCl} 6.5 \%$, and bile esculin agar containing $6 \mu \mathrm{g} / \mathrm{mL}$ vancomycin and $64 \mu \mathrm{g} / \mathrm{mL}$ ceftazidime for resistance screening. All media were kept at $37^{\circ} \mathrm{C}$ for 24 hours. Then, using the Kirby-bauer method and disks of penicillin, ampicillin, gentamicin, erythromycin, chloramphenicol, vancomycin, tetracycline, rifampicin, and clindamycin, the antibiotic resistances were determined based on CLSI protocol. In the next step, organisms that were multi-drug resistant were evaluated by using E-test strips for ampicillin, vancomycin, linezolid, imipenem, gentamicin, co-trimoxazole, and teicoplanin antibiotics in order to determine the minimum inhibitory concentration (MIC). Measurement of MIC was performed according to CLSI guidelines. After obtaining Enterococcus antibiotic susceptibility and resistance results, we used SPSS software and descriptive statistical methods (mostly frequency) to analyze these results.

\section{Results}

In this study, the mean age of patients was $73.5 \pm 1.5$ years, of which $29(54.7 \%)$ patients were men and 24 (45.3\%) were female. The percentage of samples obtained from the ICU and general wards was $24.5 \%$ and $75.5 \%$, respectively. Distribution of clinical samples were urine, 27 (53\%); sputum, 3 (5.7\%); blood, 5 (9.4\%); wounds, 7 (13.2\%); CSF, 1 (1.9\%); and bronchoalveolar lavage (BAL), 2 (3.8\%).

A total of 53 isolates of Enterococcus from clinical samples were obtained: $77.35 \%$ E. faecalis, $18.86 \%$ E. faecium, and $3.77 \%$ other species. Antibiotic susceptibility was evaluated by the disk-diffusion method and the results were as follows:

Nitrofurantoin 11 (22.9\%), vancomycin 19 (39.6\%), ampicillin $22(45.8 \%)$, penicillin $45(93.8 \%)$, tetracycline 42 (87.5\%), imipenem 26 (54.2\%), chloramphenicol 6 (12.5\%), erythromycin 46 (95.8\%), clindamycin 47 (97.9\%), reifampicin $28(58.3 \%)$, gentamicin $42(87.5 \%)$, and ciprofloxacin 45 (93.8\%).

All samples were resistant to co-trimoxazole, and in the same method, no resistance to linezolid was observed.

In the disk-diffusion method, two cases of other enterococci species were resistant to vancomycin, and the E. faecium strain was resistant to ampicillin, penicillin, ciprofloxacin, tetracycline, imipenem, erythromycin, and clindamycin. An Enterococcus species survey by the Etest method revealed 54\%, 68\%, 100\%, 93.8\%, 60.4\%, 66.7\%, $0 \%$, and $29.2 \%$ resistances to imipenem, ampicillin, cotrimoxazole, gentamicin, rifampicin, vancomycin, linezolid, and teicoplanin, respectively. Among the strains of enterococci, E. faecium $90.9 \%$ and E. faecalis $20 \%$ were resistant to vancomycin.

\section{Discussion}

Vancomycin-resistant enterococci species are considered one of the most important factors regarding nosocomial infection in susceptible patients $(7,8)$. In this study, like other studies, the prevalence of Enterococcus faecalis infection is higher than other strains (73\% vs. 23\%). Also, the resistances of E. faecalis are greater than $E$. faecium (9). Based on the results of 53 clinical specimens infected with Enterococcus, 22 (66.7\%) were resistant to vancomycin; E. faecium 20 (90.9\%), and E. faecalis 2 (9.1\%) cases, respectively. A study by Emaneini et al. in Tehran hospitals during 2006 (10) mentioned a 12\% incidence of vancomycin-resistant enterococci species. In this study, we found more incidence of resistance than in Amani and colleagues' research. This study showed that the increasing upward trend toward resistance can be attributed to the indiscriminate use of antibiotics.

A 2008 report by Feizabadi et al. in Labbafinejad, Tehran, showed that $100 \%$ of E. faecalis isolates from urine samples were susceptible to vancomycin by disk-diffusion method, whereas E. faecium samples were $71 \%$ resistant to vancomycin (11-13). The results of this study are somewhat different from the results of our study statistically, which could be due to the type of study, or the increasing trend of antibiotic resistance and resistance patterns in hospitals. Another possible reason for this difference could be related to the hospital itself, in that a teaching hospital's antibiotic prescription process is more logical $(14,15)$.

In our study, Enterococcus species by the E-test method showed the highest resistance to co-trimoxazole (100\%), gentamicin (93.8\%), and ampicillin (68\%). All E. faecium samples were resistant to gentamaicin, ampicillin, and imipenem. In Feizabad's study, E. faecium species were resistant to ampicillin (86\%), gentamicin (71\%), and imipenem (100\%).

Zhanel et al. during a 2002 study in the United States, reported that linezolid, nitrofurantoin, chloramphenicol, and synercid antibiotics are the most effective drugs for the treatment of urinary tract infections caused by a vancomycin-resistant $E$. faecium strain, while ampicillin and ciprofloxacin were the least effective (16). In our disk-diffusion method results, Enterococcus species were sensitive to linezolid in all cases, with chloramphenicol and nitrofurantoin observed as having the least impact.

In Rahimi et al. study, similar to our study, the most clinical samples of isolated E. faecium were urine samples (17). It would seem that the intensive care and nephrology wards have a greater risk of becoming infected with resistant strains. 
The studies carried out in this country and abroad have shown a pattern of increased antibiotic resistance, and we face the emergence of multi-drug resistant (MDR) strains. This could be because of prolonged hospital stays, irrational prescription of antibiotics, weakened immune systems because of underlying factors, and immune-system depressant drugs (18). Some studies have even shown that the use of growth stimulant medications in livestock farms, such as avoparcin, has increased vancomycin-resistant species of animals, humans, and the environment $(19,20)$.

\subsection{Conclusions}

It seems that the only way to deal with this changing pattern is to approach it with rational broad-spectrum drug prescriptions and treatment of Enterococcal infections. This can be done by giving proper instructions, such as having broad-spectrum drugs prescribed only by specialists in infectious diseases, and have mandatory consultations or requests required by other health groups to prescribe antibiotics and control the ascending rate of resistance. In this regard, it seems that proper health education and information are both necessary.

\section{Acknowledgments}

The authors would like to acknowledge their gratitude to Dr. Azad, who participated in data collection in Khatam-ol-Anbia hospital laboratory. We also wish to thank Miss Azar Darvishi for her kind cooperation in the statistical analysis.

\section{Footnotes}

Authors' Contribution:The core idea of this work came from Davood Yadegarynia. Zahra Arab-Mazar collected the data and acted as technical and material support.

Funding/Support:We would also like to express our gratitude to Infectious Diseases and Tropical Medicine Research Center, Shahid Beheshti University of Medical Sciences for financially supporting this research.

\section{References}

1. Low DE, Keller N, Barth A, Jones RN. Clinical prevalence, antimicrobial susceptibility, and geographic resistance patterns of enterococci: results from the SENTRY Antimicrobial Surveillance Program, 1997-1999. Clin Infect Dis. 2001;32 Suppl 2:S133-45. doi: 10.1086/320185. [PubMed:11320453]

2. Delmonico FL. Cadaver donor screening for infectious agents in solid organ transplantation. Clin Infect Dis. 2000;31(3):781-6. doi: 10.1086/314000. [PubMed:11017830]

3. Freeman RB, Giatras I, Falagas ME, Supran S, O'Connor K, Bradley J, et al. Outcome of transplantation of organs procured from bacteremic donors. Transplantation. 1999;68(8):1107-11. [PubMed: 10551637]

4. Gold HS, Moellering Jr RC. Antimicrobial-drug resistance. N Engl J Med. 1996;335(19):1445-53. doi: 10.1056/NEJM199611073351907. [PubMed: 8875923]

5. Mohanty S, Jose S, Singhal R, Sood S, Dhawan B, Das BK, et al. Species prevalence and antimicrobial susceptibility of enterococci isolated in a tertiary care hospital of North India. Southeast Asian JTrop Med Public Health. 2005;36(4):962-5. [PubMed:16295552]

6. Sader HS, Flamm RK, Jones RN. Antimicrobial activity of daptomycin tested against Gram-positive pathogens collected in Europe, Latin America, and selected countries in the Asia-Pacific Region (2011). Diagn Microbiol Infect Dis. 2013;75(4):417-22. doi: 10.1016/j.diagmicrobio.2013.01.001. [PubMed: 23514757]

7. Rolston KV, Kapadia M, Tarrand J, Coyle E, Prince RA. Spectrum of gram-positive bacteraemia and in vitro activities of daptomycin, linezolid and vancomycin against organisms isolated from cancer patients. Int J Antimicrob Agents. 2013;41(6):516-20. doi: 10.1016/j.ijantimicag.2013.01.014. [PubMed: 23481658]

8. Bauer AW, Kirby WM, Sherris JC, Turck M. Antibiotic susceptibility testing by a standardized single disk method. Am J Clin Pathol. 1966;45(4):493-6. [PubMed: 5325707]

9. Huycke MM, Sahm DF, Gilmore MS. Multiple-drug resistant enterococci: the nature of the problem and an agenda for the future. Emerg Infect Dis. 1998;4(2):239-49. doi: 10.3201/ eid0402.980211. [PubMed: 9621194]

10. Emaneini M, Aligholi M, Aminshahi M. Characterization of glycopeptides, aminoglycosides and macrolide resistance among Enterococcus faecalis and Enterococcus faecium isolates from hospitals in Tehran. Pol J Microbiol. 2008;57(2):173-8. [PubMed: 18646406]

11. Feizabadi MM, Sayadi S, Shokrzadeh L, Parvin M, Yadegarynia D. Increase in prevalence of vancomycin resistant isolates of Enterococcous faecium at Labbafinejad hospital. Arch Clin Infect Dis. 2008;3(2).

12. Lozano C, Gonzalez-Barrio D, Garcia JT, Ceballos S, Olea PP, RuizFons F, et al. Detection of vancomycin-resistant Enterococcus faecalis ST6-vanB2 and E. faecium ST915-vanA in faecal samples of wild Rattus rattus in Spain. Vet Microbiol. 2015;177(1-2):168-74. doi: 10.1016/j.vetmic.2015.02.025. [PubMed: 25795517]

13. Smith JR, Barber KE, Raut A, Rybak MJ. beta-Lactams enhance daptomycin activity against vancomycin-resistant Enterococcus faecalis and Enterococcus faecium in in vitro pharmacokinetic/pharmacodynamic models. Antimicrob Agents Chemother. 2015;59(5):2842-8. doi: 10.1128/AAC.00053-15. [PubMed: 25753639]

14. Khalili H, Dashti-Khavidaki S, Karimzadeh I, Jafari S, Abdollahi A, Shahidi MR, et al. Changes in 4-year antimicrobial resistance pattern of gram-positive bacteria at the main referral teaching hospital, Tehran, Iran. Acta Med Iran. 2012;50(7):493-504. [PubMed: 22930383]

15. Sreeja S, Babu PRS, Prathab AG. The prevalence and the characterization of the enterococcus species from various clinical samples in a tertiary care hospital. J Clin Diagn Res. 2012;6(9):1486-8. doi:10.7860/JCDR/2012/4560.2539. [PubMed: 23285436]

16. Zhanel GG, Laing NM, Nichol KA, Palatnick LP, Noreddin A, Hisanaga T, et al. Antibiotic activity against urinary tract infection (UTI) isolates of vancomycin-resistant enterococci (VRE): results from the 2002 North American Vancomycin Resistant Enterococci Susceptibility Study (NAVRESS). J Antimicrob Chemother. 2003;52(3):382-8. doi:10.1093/jac/dkg352. [PubMed:12888592]

17. Rahimi F, Talebi M, Saifi M, Pourshafie MR. Distribution of enterococcal species and detection of vancomycin resistance genes by multiplex PCR in Tehran sewage. Iran Biomed J. 2007;11(3):161-7. [PubMed: 18051776]

18. Mikalsen T, Pedersen T, Willems R, Coque TM, Werner G, Sadowy $\mathrm{E}$, et al. Investigating the mobilome in clinically important lineages of Enterococcus faecium and Enterococcus faecalis. BMC Genomics. 2015;16:282. doi: 10.1186/s12864-015-1407-6. [PubMed: 25885771]

19. Wegener HC, Aarestrup FM, Jensen LB, Hammerum AM, Bager F. Use of antimicrobial growth promoters in food animals and Enterococcus faecium resistance to therapeutic antimicrobial drugs in Europe. Emerg Infect Dis. 1999;5(3):329-35. doi: 10.3201/ eid0503.990303. [PubMed:10341169]

20. Mourand G, Jouy E, Bougeard S, Dheilly A, Kerouanton A, Zeitouni $S$, et al. Experimental study of the impact of antimicrobial treatments on Campylobacter, Enterococcus and PCR-capillary electrophoresis single-strand conformation polymorphism profiles of the gut microbiota of chickens. J Med Microbiol. 2014;63(Pt 11):1552-60. doi:10.1099/jmm.0.074476-0. [PubMed: 25142966] 